\title{
New Image Processing Methodology for Noisy-Blurred Images
}

\author{
Woosang Jeon ${ }^{1^{*}}$ and Kun-Hee Han $^{2}$ \\ ${ }^{1}$ WSConsulting \\ ${ }^{2}$ Division of Information \& Communication Engineering, Baekseok University \\ 잡음으로 훼손된 영상에 대한 새로운 영상처리방법론 \\ 전우상 $^{1^{*}}$, 한군희 $^{2}$ \\ ${ }^{1} \mathrm{WS}$ 컨설팅, ${ }^{2}$ 백석대학교 정보통신학부
}

\begin{abstract}
In this paper, a iterative image restoration method is proposed to restore for noisy-blurred images. In conventional method, regularization is usually applied to all over the without considering the local characteristics of image. As a result, ringing artifacts appear in edge regions and the noise amplification is introduced in flat regions. To solvethis problem we proposed an adaptive regularization iterative restoration using directional regularization operator considering edges in four directions and the regularization operator with no direction for flat regions. We verified that the proposed methods showed better results in the suppression of the noise amplification in flat regions, and introduced less ringing artifacts in edge regions. As a result it showed visually better image and improved better ISNR further than the conventional methods.
\end{abstract}

요 약 본 논문은 블러(blur)되고 잡음이 추가되어 훼손된 영상에 대한 복구를 하기 위해 반복영상처리를 사용한 새 로운 방식을 제안한다. 전통적인 복구방법은 영상의 지역적인 특성을 고려하지 않고 일률적으로 복구방법을 적용하여 복구한다. 그 결과로서 에지에서는 인공잡음이 나타나고 평면에서는 잡음이 증폭되는 특성이 나타난다. 이러한 문제 를 해결하기 위한 방법론으로 에지 방향에 대한 방향성을 추적하여 복구를 시도하는 것을 제안한다. 기존의 방법과 제안된 방법론의 비교를 통해 제안된 방법론의 우월성을 객관적으로 나타내고자 한다.

Key Words : Edge Direction Information, Direction of Mask

\section{Introduction}

Because of the imperfection in image processing system, most images are degraded in the course of formation and recording of images. Generally there are two kinds of degradation components, blur and noise. We can model the degraded image as a formation which original image is blurred with point spreading function, and mixed with additive noise.

In the restoration of noisy-blurred image, we have trade-off problem between smoothing the noise and restoration of the edge region. While the noise is smoothed, the edge or details will corrupt. On the other hand, restoring the edge will amplify the noise more clear[1].

Especially, applying regularization uniformly all over the image without considering local characteristics makes it inevitable to cause ripple artifact in the edge regions and noise amplification in smooth regions showing some visual nuisances[2-4].

To solve this problem we proposed an new regularization restoration method using directional regularization operator considering edges in four directions and the regularization operator with no direction for flat regions. We verified that the proposed method showed better results in the suppression of the 
noise amplification in flat regions, and introduced less ringing artifacts in edge regions. In Section 2, we introduce a general conception of Tikhonov-Miller regularization. Section 3 proposes a new regularization operator considering the directional information and adaptive acceleration parameter and adaptive regularization parameter. Experimental results are presented in Section 4, and conclusions in Section 5.

\section{Tikhonov-Miller Regularization}

In most cases, the image degradation process can be modeled by a out-of-focus blur and an additive white Gaussian noise process [2], that is

$$
g=H f+n
$$

, where the lexicographically ordered vectors $g, f, n$ represent the noisy blurred image, the original image and the additive noise, respectively. The matrix $H$ represents point spread function.

Based on the image degradation model of (1) there have been a number of approaches developed to restore the original image $f$, given the observed image $g, H$ and some knowledge about the noise $n$. Among those are the set theoretic approach presented in [3], which have Miller's regularization approach.

The concept of regularization is to define a criterion to select an approximate solution from a set of feasible solutions [4-7],

$$
\Phi(\hat{f})=\|g-H \hat{f}\|^{2}+\alpha\|C \hat{f}\|^{2}
$$

Where $\mathrm{C}$ is the regularization operator and $\alpha$ is regularization parameter controlling the contribution of the terms $\|g-H \hat{f}\|^{2}$ and $\|C \hat{f}\|^{2}$. The restored image is the vector $\hat{f}$, which minimizes the object function $\Phi(\hat{f})$. If bounds of $\|g-H \hat{f}\|^{2}$ and $\|C \hat{f}\|^{2}$, denoted respectively by $\varepsilon^{2}$ and $E^{2}$, are known, then $\alpha$ is given as $(\varepsilon / E)^{2}$ [3].

We need to minimize the object function with respect to $\hat{f}$ and this minimization is straightforward and leads to the following solution:

$$
\hat{f}=\left(H^{T} H+\alpha C^{T} C\right)^{-1} H^{T} g .
$$

When $\left(H^{T} H+\alpha C^{T} C\right)^{-1}$ has any ill-condition or singular point it is impossible to restore or it needs a lot of calculation. An alternative to the direct computation of $\hat{f}$ in (3) is the iterative implementation. The way to solve this problem is the iterative Tikhonov-Miller regularization restoration method that applies regularization theory into an existing iteration processing method. If we rearrange (3) applying successive substitution method, you can get iteration processing method [4] as

$$
\hat{f}_{k+1}=\hat{f}_{k}+\beta\left[H^{T} g-\left(H^{T} H+\alpha C^{T} C\right) \hat{f}_{k}\right]
$$

, where $\beta$ is used to guarantee convergence as a accelerator parameter.

\section{Definition of the regularization operator}

\subsection{Extraction of Edge direction}

There are several methods to extract the edge direction information from the blurred image that contains noise. In this case, we extracted the edge directional information using template matching method with prewitt operator to reduce the effect of noise. In Fig.1, using the eight type mask that faces to direction of edge, we decided direction of mask $d_{i, j}$ that is explaining the value of maximum that came from iteration of each pixel. When the case that pixel was decided edge, we decided edge direction information, $\theta(i, j)$

$$
d_{i, j}=\operatorname{Max}\left[\left|\sum_{k=-1 l=-1}^{1} \sum_{k, l}^{1}(\theta) x_{i-k, j-l}\right|\right]
$$

$\theta=\{0,45,90,135\}$ is the angle which points direction and $P_{k, l}(\theta)$ is direction differentiational operator.

Decision of the edge rigion and flat region of each pixels decided that compare with threshold and local 
variance value of pixel. The threshold is value of variance of noise came from flat region as each iteration times. Using (5), we found edge direction information like below.

$$
\theta(i, j)= \begin{cases}d_{i, j}, & \sigma^{2} f_{k}(i, j) \geq T(\text { edge region }) \\ 360^{\circ}, & \sigma_{f_{k}(i, j)}^{2}<T(\text { flat region })\end{cases}
$$

$\sigma_{f_{k}(i, j)}^{2}$ is variance value of local and flat region considered $360^{\circ}$

\subsection{Parameters}

It is different that the amount of noisy according to local property of pixels even though blurring is adopted uniformly to total image. In other words, in small variance flat region, noisy error is shown little, and in large variance edge region, noisy shown largely. If you doing iterative restoration using this fact, it is better to use adaptive acceleration parameter than uniformly acceleration parameter. And then total convergence ration is fast, especially import convergence of edge region is getting faster. Also we can have effective restoration and resolution increasing better than conventional method. In this paper, we proposed adaptive acceleration parameter (7).

$$
\beta_{k}(i, j)=2 \cdot \frac{\sigma^{2} f_{k}(i, j)}{\sigma_{n}^{2}+\sigma_{f_{k}(i, j)}}
$$

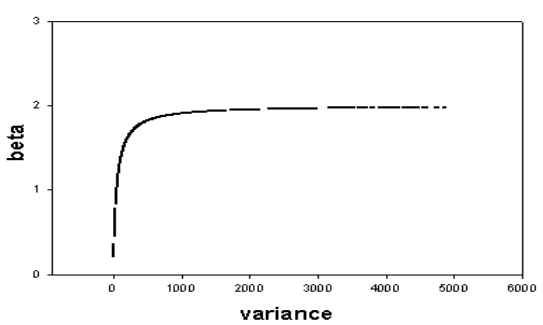

[Fig. 1] ratio of acceleration parameter for variance

$$
\sigma_{n}^{2}, \sigma^{2} f_{k}(i, j) \text { is each variance of noise and variance of }
$$
restored image. When the variance of restored image is less than variance value of noise $\sigma^{2}{ }_{n}$, adaptive acceleration parameter $\beta_{k}(i, j)$ closed to 0 .. And when the variance of restored image is more than variance of noise $\sigma^{2}{ }_{n}$, it also closed to 2 . Therefore adaptive acceleration parameter $\beta_{k}(i, j)$ has a value between $0 \sim 2$ according to variance $\sigma_{n}^{2}, \sigma_{f_{k}(i, j)}^{2}$. In the right of (7), 2 is used to fast convergence ratio and satisfy convergence condition.

In this paper calculated regularization parameter $\alpha$ like below. The regularization parameter $\alpha$ can be written as :

$$
\alpha=\left(\frac{\varepsilon}{E}\right)^{2}=\frac{\|g-H \hat{f}\|^{2}}{\|C \hat{f}\|^{2}}
$$

Ultimately, the regularization parameter $\alpha$ is inversely proportional with SNR. Considering this fact, In this paper, we can modify the adaptive regularization parameter as[1] :

$$
\alpha_{k}(i, j)=\frac{1}{1+\frac{\sigma_{f_{k}(i, j)}^{2}}{\sigma^{2}{ }_{n}}}
$$

The adaptive regularization parameter $\alpha_{k}(i, j)$ is close to 0 when the case variance of restored image is larger than variance of noise. If situation is opposite, it closed to 1 . If variance of local of noise and restored image is equal, it's value 0.5 .

\subsection{Directional operators}

In the restoration of noisy-blurred images, we have a trade-off problem between smoothing the noise and restoring the edge regions. While the noise is smoothed, the edges or detail will become corrupt. On the other hand, restoring the edges will amplify the noise making it more prominent.

One of the biggest problem to restore the noisy-blurred image is smoothing of edge region and suppressing of noise. Conventional methods are regularization restoration that does not consider the local property. Especially when we used Laplacian operator with regularization operator, it caused ripple noise in edge region. also it did not satisfy visually.

In this paper, to solve these problems, we suggested directional regularization operator as Fig. 2. It divided two, flat region and 4-direction edge region. This directional regularization operator restored efficiently and removed the ripple noise in the edge regions. 


$$
\left[\begin{array}{ccc}
0 & 0 & 0 \\
-0.5 & 1 & -0.5 \\
0 & 0 & 0
\end{array}\right] \quad\left[\begin{array}{ccc}
0 & 0 & -0.5 \\
0 & 1 & 0 \\
-0.5 & 0 & 0
\end{array}\right] \quad\left[\begin{array}{ccc}
0 & -0.5 & 0 \\
0 & 1 & 0 \\
0 & -0.5 & 0
\end{array}\right]
$$
(a) $0^{\circ}$
(b) $45^{\circ}$
(c) $90^{\circ}$

$$
\left[\begin{array}{ccc}
-0.5 & 0 & 0 \\
0 & 1 & 0 \\
0 & 0 & -0.5
\end{array}\right] \quad\left[\begin{array}{ccc}
0 & -0.25 & 0 \\
-0.5 & 1 & -0.5 \\
0 & -0.25 & 0
\end{array}\right]
$$
(d) $135^{\circ}$
(e) $360^{\circ}$

[Fig. 2] directional operators

In this paper proposed new regularization restoration method (4) using adaptive acceleration parameter (7) and adaptive regularization parameter (9). In this paper proposed also directional regularization operator as regularization operator. This method extracts local property or directional information in the flat regions and the edge regions. In the flat regions, less acceleration parameter stable convergence ratio and suppressed amplification of noise using conventional Laplacian operator. In the edge region, convergence ratio is getting faster by large accelerator parameter and it improves not only resolution of edge region but also reduce the ripple noise using directional regularization operator.

\section{Experimental Results}

The standard image used in experiment is 256 by 256 size "Lena" image and "Camera man"image. Noisy image is added image with pseudo Gaussian white noise that contains BSNR(Blurred SNR) of 20dB and blurred image by motion blur. In this case BSNR is like below

$$
B S N R=\frac{\text { variance of the blurred image }}{\text { variance of the additive noise }}=\frac{\sigma^{2}{ }_{\mathrm{Hf}}}{\sigma^{2}{ }_{n}}
$$

Here, $\sigma^{2}{ }_{n}$ is the variance of pseudo-Gaussian white noise and $\sigma_{H f}^{2}$ denotes the variance of the blurred image.

The performance of the restoration algorithm is evaluated by measuring the improved SNR defined by :

$$
I S N R=10 \log \frac{\sum_{(i, j) \in I}(g(i, j)-f(i, j))^{2}}{\sum_{(i, j) \in I}\left(f(i, j)-\hat{f}_{k}(i, j)\right)^{2}}
$$

This paper experiments analyzed with three methods. First method is conventional regularization restoration. It fixed $\alpha=0.5, \beta=1.0$ and used Laplacian operator as regularization operator. Second method is proposed method 1. It used directional regularization operator as regularization operator and fixed $\alpha=0.5, \beta=1.0$. Third method is proposed ethod 2. It used adaptive accelerator parameter, adaptive regularization parameter and directional regularization operator.

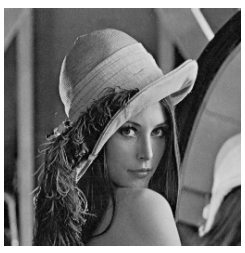

(a) Original image

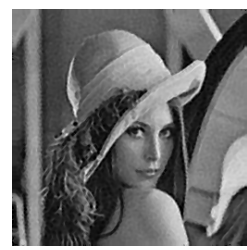

(c) Laplacian

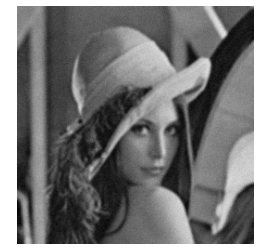

(b) Degradation Image

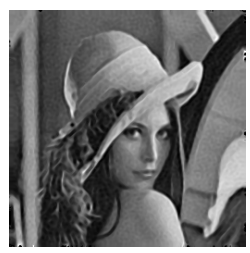

(d) directional using $\alpha=0.5, \beta=1.0$

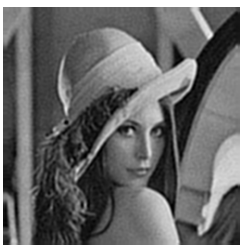

(e) Proposed directional regularization operator using

$$
\alpha_{k}(\cdot, \cdot), \beta_{k}(\cdot, \cdot)
$$

[Fig. 3] restored images for "Lena"

In Fig. 3 and Fig. 4 showed the restoration results for conventional method and suggested methods. Each (a) is original image and (b) is noisy-blurred image. (c) was result of experimental with Laplacian regularization operator to the whole image in conventional method. We can find lots of blurring in edge region and noise also remain in the flat region. (d) used directional regularization operator that is suggested first method in this paper. (e) used directional regularization operator that is suggested second method in this paper.

As a result, the suggested methods improved the 
resolution better than the conventional method. Resolution is improved in edge region and noise also reduced. Ripple noise also reduced a lot in the edge part and noise is reduced in the flat region of background.

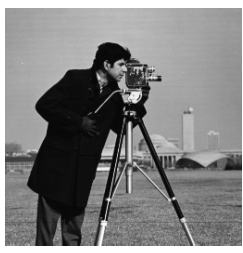

(a) Original image

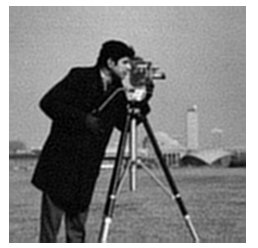

(c) Laplacian

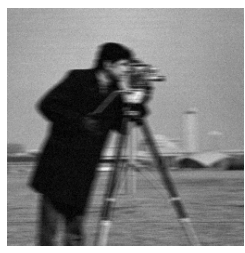

(b) Degradation Image

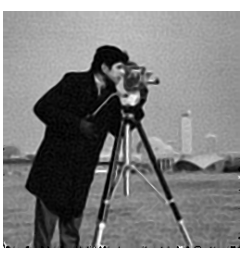

(d) Proposed using

$$
\alpha=0.5, \beta=1.0
$$

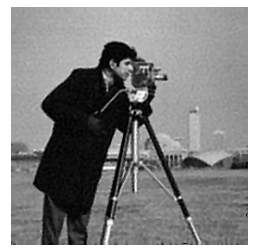

(e) Proposed directional regularization operator using $\alpha_{k}(\cdot, \cdot), \beta_{k}(\cdot, \cdot)$

[Fig. 4] restored images for "Camera man
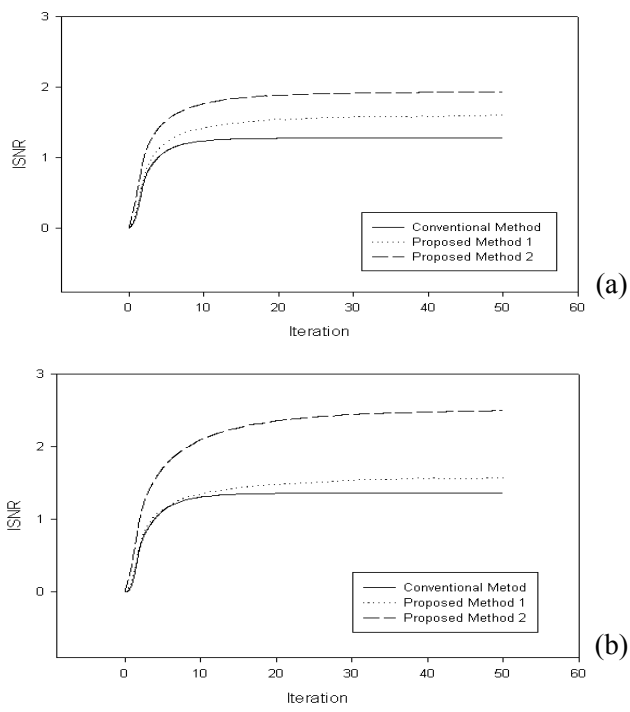

[Fig. 5] ISNRs for images of "Lena" and "Camera man"
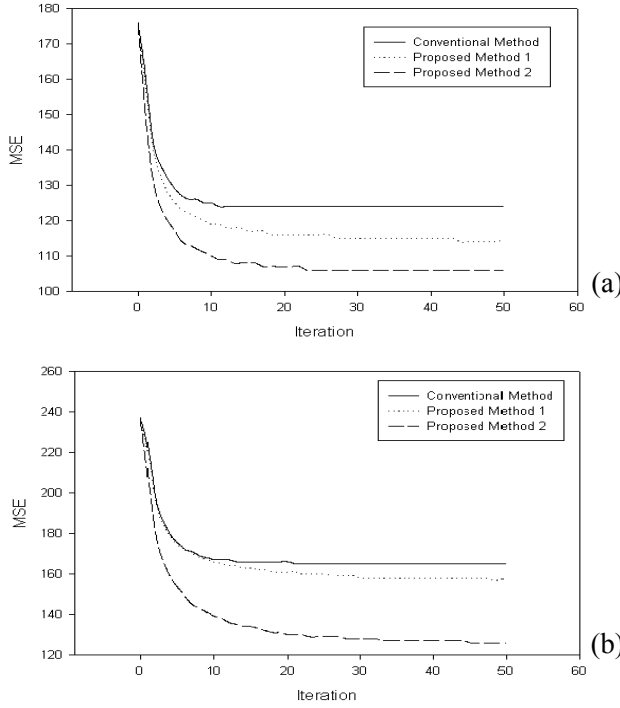

[Fig. 6] MSE for images of "Lena" and "Camera man"

The corresponding ISNRs for several methods were shown versus the iteration number in Fig. 5. In Fig. 5(a), ISNRs for conventional method were $0.19,1.24,1.28$, $1.28,1.28,1.28 \mathrm{~dB}$ at each of iteration of $1,10,20,30$, 40, 50, where ISNRs of proposed method 1 were 0.25 , $1.42,1.54,1.57,1.59,1.60 \mathrm{~dB}$, where ISNRs of proposed method 2 were $0.46,1.77,1.89,1.92,1.93,1.93 \mathrm{~dB}$. Proposed method improved each $0.32,0.65 \mathrm{~dB}$ compare with conventional method in "Lena" image.

In Fig. 5(b), ISNRs for conventional method were $0.13,1.31,1.36,1.36,1.36,1.36 \mathrm{~dB}$ at each of iteration of $1,10,20,30,40,50$, where ISNRs of proposed method 1 were $0.18,1.35,1.48,1.54,1.57,1.57 \mathrm{~dB}$, where ISNRs of proposed method 2 were $0.43, .210,2.36,2.44,2.48$, $2.50 \mathrm{~dB}$. Proposed method improved each $0.21,1.14 \mathrm{~dB}$ compare with conventional method in "Camera man" image. And also the improvement ISNR of the method 2 is less than "Lena" image. Because the edge region of "Camera man"image is less than "Lena" image.

The suggested methods in this paper make the convergence ratio better than the conventional method when iterated each time and as well as improved ISNRs. When the different images adopted methods of suggested, we can get similar result as "Lena" image and "Camera man" image.

As a result, adaptive regularization restoration methods considering direct information of suggested in this paper 
improved the resolution better than conventional method, reduce the noise in flat region, ripple noise also reduce in the edge region and there is improved effectiveness in the aspect of convergence.

\section{Conclusion}

The conventional restoration method didn't considered local property of image and done regularization to the whole image. It can't escape amplification of noise in flat region. Especially when we restoring the edge region using Laplacian operator as regularization operator makes ripple noise, so they can't have satisfying result. Toimprove this problem, in this paper we suggested new regularization restoration method operate according to the directional properties of flat region and edge region. It divided image into flat region and edge region that has 4-direction. And then we used regularization operator considered edge direction. The suggested methods got experiment improved smoothing of noise in flat region more than conventional method. By reducing ripple noise of improvement of edge direction or edge directional information, we can get more satisfying result.

\section{References}

[1] Woosang Jeon, "Image Restoration using Dual Adaptive Regularization Operators", in Proc. IEEE Int. Conf. ICPR, 2000, pp. 49-52.

[2] J. S. LIM, Two-Dimensional Signal and Image Processing, Prentice-Hall, 1990.

[3] A. K. Katsaggelos, J. Biemond, R. W. Schafer and R. M. Mersereau, "A Regularized Iterative Image Restoration Algorithm", IEEE Trans. Signal Processing, Vol. 39, April 1991, No. 4, pp.914-929.

[4] Reginald L. Lagendijk, and Jan Biemond, Iterative Identification and Restoration of Image, Kluwer Academic Publishers, 1991.

[5] S. Kawata and Y, Ichioka, "Iterative Image Restoration for linearly Degraded Image. I .Basis," J. Opt. Soc. Amer., Vol.70, No.7, pp.762-768, July, 1980

[6] Aggelos K. Katsaggelos, "Iterative Image Restoration Algorithms", Optical Eng., Vol. 28, No. 7, pp. 735-748, July 1989.

[7] R. L. Lagendijk , J. Biemond and D.E. Boekee, "Regularized Iterative Image restoration with Ringing Reduction," IEEE trans. ASSP, Vol.36, No.12, pp.1874-1888, December, 1988.

\section{Woosang Jeon}

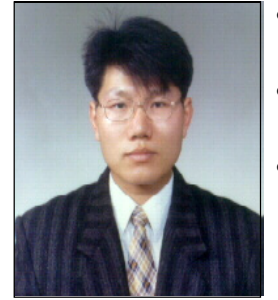

- $2004 \sim$ current : Fellow Consultant, TIPA IMS Assessment \& Standardization Committee, PM \& Leadership Educator, WSConsulting CEO

$<$ Research Interests $>$

Business Innovation, RFID, USN, CISA, KCISA, Digital Signal Processing, Pattern Recognition, Network, DB, Embedded System

\section{Kun-Hee Han}

[Life member]

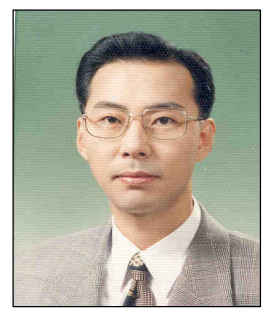

- Aug. $2008 \sim$ current : Baekseok University, Division of Information \& Communication Engineering, professor

$<$ Research Interests $>$

WSN, RFID, Wireless LAN security, Pattern Recognition 